\title{
CONDICIONAMENTO FISIOLÓGICO DE SEMENTES DE PEPINO E GERMINAÇÃO SOB DIFERENTES TEMPERATURAS ${ }^{1}$
}

\author{
LIANA BAPTISTA DE LIMA²; JÚLIO MARCOS FILHO³
}

\begin{abstract}
RESUMO - O condicionamento fisiológico é uma técnica utilizada para a embebição controlada de sementes e tem como benefícios, aumentar a rapidez e uniformidade na emergência de plântulas e a tolerância das sementes a condições ambientais adversas. O presente trabalho teve como objetivo estudar procedimentos para o condicionamento fisiológico e verificar o desempenho das sementes quando colocadas para germinar sob diferentes temperaturas. Foram utilizados lotes de sementes dos cultivares Safira e Jóia, testando-se o osmocondicionamento a -0,1MPa e-0,2MPa até que as sementes do cv. Safira atingissem o teor de água de $29 \%$, e do cv. Jóia, $32 \%$. Os efeitos do condicionamento foram avaliados pela porcentagem e velocidade de germinação a $15,20,25,30$ e $35^{\circ} \mathrm{C}$. Verificouse que o osmocondicionamento em papel embebido em solução de PEG -0,2MPa é benéfico ao desempenho de sementes de pepino, favorecendo a velocidade de germinação para a maioria dos lotes e temperaturas testados, com resultados mais expressivos em lotes de menor vigor expostos a temperaturas subótimas.
\end{abstract}

Termos para indexação: fisiologia de sementes, osmocondicionamento, desempenho.

\section{CUCUMBER (Cucumis sativus) SEED PRIMING METHODS AND GERMINATION AT DIFFERENT TEMPERATURES}

\begin{abstract}
Seed priming is a technique that involves the controlled hydration of seeds and has beneficial effects such as increased velocity and uniformity of seedling emergence and increased seed tolerance to unfavorable environmental conditions. We defined procedures for seed priming and verified seed performance at different temperatures using seed lots of $C$. Sativus cultivars Safira and Joia submitted to osmopriming ( -0.1 and $-0.2 \mathrm{MPa}$ ) to a seed moisture content of $29 \% \mathrm{for} \mathrm{cv}$. Safira and $32 \%$ for cv. Joia. The effects of osmoconditioning were evaluated by percentage and speed of germination at $15,20,25,30$ and $35^{\circ} \mathrm{C}$. The results indicated that seed osmopriming between sheets of paper towel moistened with -0.2 MPa polyethylene glycol (PEG) solution is efficient for cucumber seeds, affecting the speed of seed germination for almost every seed lot and temperature tested. Results were particularly good when seed from low-vigor lots were exposed to sub-optimal temperatures.
\end{abstract}

Index terms: osmopriming, polyethylene glycol (PEG), performance, seed physiology.

${ }^{1}$ Submetido em 07/04/2009. Aceito para a publicação em 21/08/2009. Parte da Tese de Doutorado do primeiro autor apresentada à USP/ESALQ. Doutorado realizado com bolsa FAPESP.

${ }^{2}$ Eng.Agr. Dr., Bolsista CNPq Programa DCR/Fundect, Departamento de Biologia UFMS, CP 549, CEP 79070-900, Campo Grande, MS, lianablima@gmail.com

${ }^{3}$ Eng. Agr. Dr. Professor Titular, Departamento de Produção Vegetal USP/ESALQ, CP 09, 13418-900, Piracicaba, SP. Bolsista CNPq, jmarcos@esalq.usp.br 


\section{INTRODUÇÃO}

A utilização de novas cultivares de hortaliças, especialmente de híbridos, tem colaborado para avanços na tecnologia de produção e cultivo, como, por exemplo, o cultivo protegido, o uso do transplante de mudas para obtenção do estande adequado no campo em substituição à semeadura direta, para algumas espécies. Sendo esse um dos primeiros passos para obtenção de um cultivo economicamente compensador, a produção de mudas em bandejas demanda alta eficiência no desempenho das sementes, com capacidade para gerar plântulas vigorosas e uniformes no menor período de tempo.

A ocorrência de condições ambientais adversas após a semeadura não pode ser prevista ou mesmo evitada, mas contribui para a desuniformidade e redução do estande, tanto em bandejas para produção de mudas quanto no cultivo por meio de semeadura direta, com possíveis prejuízos ao produtor. Desta maneira, em culturas de hortaliças, cujas sementes possuem alto valor comercial, o uso de procedimentos especiais, como o condicionamento fisiológico, pode favorecer o desempenho das sementes.

O condicionamento fisiológico é uma técnica utilizada para a embebição controlada de sementes, permitindo a ativação dos processos metabólicos da germinação, sem que ocorra emissão da raiz primária (Heydecker et al., 1973). Dentre os benefícios promovidos por esse tratamento, destacam-se a rapidez e uniformidade na emergência de plântulas (Knypl e Khan, 1981; Marcos Filho e Kikuti, 2008) e a tolerância das sementes a condições ambientais menos favoráveis (Trigo e Trigo, 1999; Nascimento, 2005; Pereira et al., 2009).

No entanto, esses efeitos são influenciados por muitas variáveis, como método para o condicionamento, período e temperatura durante o tratamento, espécie e cultivar e potencial fisiológico inicial do lote utilizado, de forma que não há um procedimento único para o condicionamento de sementes de diferentes espécies (Welbaum et al., 1998).

Assim, várias espécies têm sido estudadas quanto à utilização desta técnica, como: alface (Fessel et al., 2002), berinjela (Fanan e Novembre, 2007; Nascimento e Lima, 2008), brócolos (Jett et al., 1996), cebola (Caseiro et al., 2004), cenoura (Carneiro et al., 1999; Pereira et al., 2009), couve-flor (Marcos Filho e Kikuti, 2008), milho-doce (Parera e Cantliffe, 1991), quiabo (Dias et al., 1999), melão (Nascimento, 2003); pepino (Passam et al., 1989); pimentão (Roveri José et al., 2000) e tomate (Andreoli e Khan, 1999; Rossetto et al., 2002).
No entanto, a disponibilidade de informações na literatura com relação à aplicação da técnica em sementes de pepino e seus efeitos sobre o desempenho das sementes quando expostas a temperaturas adversas durante a germinação é relativamente escassa, havendo necessidade de aprofundar os estudos para essa espécie.

Desta maneira, no presente trabalho objetivou-se: verificar se o método do osmocondicionamento pode constituir alternativa viável para o condicionamento fisiológico de sementes de pepino e verificar os efeitos deste tratamento sobre a germinação em diferentes temperaturas.

\section{MATERIAL E MÉTODOS}

O presente trabalho foi conduzido no Laboratório de Análise de Sementes da Escola Superior de Agricultura "Luiz de Queiroz" da Universidade de São Paulo (USP/ESALQ), em Piracicaba - SP. Foram utilizados seis lotes de sementes de pepino, três do cultivar Safira e três do cultivar Jóia, com diferentes potenciais fisiológicos. Durante o período experimental, as sementes permaneceram embaladas em sacos de papel multifoliado e armazenadas em condições controladas $\left(20^{\circ} \mathrm{C}\right.$ e $50 \%$ UR).

Os lotes foram avaliados inicialmente mediante determinações do teor de água, porcentagem e velocidade de germinação, porcentagem e velocidade de emergência de plântulas, condutividade elétrica e envelhecimento acelerado.

Teor de água das sementes: determinado pelo método da estufa a $105 \pm 3{ }^{\circ} \mathrm{C}$, por 24 horas, utilizando duas repetições de 1g para cada tratamento (Brasil, 1992), com resultados expressos em porcentagem (base úmida).

Germinação: Quatro repetições de 50 sementes para cada lote foram distribuídas em rolos de papel toalha umedecidos com quantidade de água equivalente a 2,0 vezes o peso do substrato seco e colocadas para germinar a $25{ }^{\circ} \mathrm{C}$. A contagem das plântulas normais foi diária, determinando-se a velocidade de germinação (Maguire, 1962) e a porcentagem de plântulas normais aos 4 e 8 dias após semeadura (Brasil, 1992).

Emergência de plântulas: quatro repetições de 50 sementes para cada lote foram distribuídas em bandejas de poliestireno de 200 células contendo substrato Plantmax ${ }^{\circledR}$. As bandejas foram mantidas em ambiente protegido, sob temperatura média de $25{ }^{\circ} \mathrm{C}$; a contagem de plântulas emergidas foi realizada diariamente determinando-se a velocidade de emergência (Maguire, 1962) e a porcentagem de plântulas no oitavo dia após a semeadura. 
Condutividade elétrica: quatro repetições de 50 sementes foram pesadas com precisão de $0,0001 \mathrm{~g}$, colocadas em copos de plástico com capacidade para $200 \mathrm{ml}$, contendo $100 \mathrm{ml}$ de água destilada e embebidas a $25^{\circ} \mathrm{C}$. A condutividade elétrica da solução de embebição foi determinada após 16 horas, e os resultados foram expressos em $\mu$ mho. $\mathrm{cm}^{-1} . \mathrm{g}^{-1} \mathrm{de}$ sementes.

Envelhecimento acelerado: conduzido de acordo com o método com solução saturada de cloreto de sódio, distribuindo-se sementes de cada amostra formando camada única sobre a superfície de tela metálica, suspensa no interior de caixa de plástico $(11 \times 11 \times 13 \mathrm{~cm})$, contendo $40 \mathrm{ml}$ de solução saturada de cloreto de sódio. As caixas foram mantidas em câmara jaquetada de água a $41{ }^{\circ} \mathrm{C}$, durante 96 horas. Após o envelhecimento, foi conduzido teste de germinação computando-se a porcentagem de plântulas normais aos 4 dias após a semeadura.

Osmocondicionamento: o estudo dos procedimentos para o condicionamento fisiológico foi realizado comparando-se os efeitos do osmocondicionamento realizado em papel embebido em solução de PEG 6000 a $-01 \mathrm{MPa}$ e a $-0,2 \mathrm{MPa}$. Duas camadas de três folhas de papel toalha foram embebidas com quantidade de solução equivalente a 2,5 vezes o peso do papel em água, onde as sementes foram distribuídas, permanecendo a $25{ }^{\circ} \mathrm{C}$ até atingir o teor de água final, sendo $29 \%$ para as sementes do cv. Safira e 32\% para as sementes do cv. Jóia (esses teores foram definidos com base na determinação da marcha de absorção de água pelas sementes; dados não apresentados). Atingidos os teores de água desejados, as sementes foram retiradas do papel-toalha para determinação do teor de água.
Os efeitos do condicionamento fisiológico foram avaliados por porcentagem e velocidade de germinação a 15, 20, 25, 30 e $35^{\circ} \mathrm{C}$, utilizando-se a mesma metodologia do teste de germinação descrita anteriormente, com variação da temperatura.

Procedimento estatístico: utilizou-se o delineamento em blocos inteiramente casualizados com quatro repetições. Os dados foram submetidos à análise de variância separadamente para cada cultivar e após o condicionamento para cada lote e temperatura de germinação as médias foram comparadas pelo teste de Tukey $(\mathrm{p} \leq 0,05)$.

\section{RESULTADOS E DISCUSSÃO}

\section{Avaliação inicial dos lotes}

Os lotes de sementes dos cultivares Safira e Jóia foram caracterizados por meio da determinação do teor de água, da porcentagem e velocidade de germinação e de emergência de plântulas, da condutividade elétrica e do envelhecimento acelerado (Tabela 1). Observa-se que houve efeito significativo para os lotes, que apresentavam diferenças quanto ao vigor. Assim, a porcentagem e velocidade de germinação, a velocidade de emergência e os resultados do teste de envelhecimento acelerado, identificaram o lote 3 do cv. Safira como de menor vigor e o lote 2 o de maior vigor. Paralelamente, no teste de condutividade elétrica e na porcentagem de germinação não foram observadas diferenças entre os lotes do cultivar Jóia. Os demais testes acusaram a inferioridade do desempenho do lote $\mathrm{C}$ e a superioridade do lote A (Tabela 1).

TABELA 1. Dados médios referentes ao teor de água (TA), porcentagem e velocidade de germinação (índice), porcentagem e velocidade de emergência de plântulas (índice), condutividade elétrica $\left(\mu \mathrm{mho} . \mathrm{cm}^{-1} \cdot \mathrm{g}^{-1}\right) \mathrm{e}$ envelhecimento acelerado (\%), de lotes de sementes dos cultivares Safira e Jóia.

\begin{tabular}{|c|c|c|c|c|c|c|c|c|}
\hline Cultivar & Lotes & TA & \multicolumn{2}{|c|}{ Germinação } & \multicolumn{2}{|c|}{ Emergência } & Cond. elét. & Env. acel. \\
\hline \multirow{5}{*}{ Safira } & & $\ldots \ldots \% \ldots$ & $\ldots . . \% \ldots$ & Índice & $\ldots . . \% \ldots$ & índice & $\mu \mathrm{mho} . \mathrm{cm}^{-1} \cdot \mathrm{g}^{-1}$ & ......\% $\% \ldots \ldots$ \\
\hline & 1 & 6,3 & $100 \mathrm{a}$ & $37,8 \mathrm{~b}$ & $98 \mathrm{a}$ & $31,2 \mathrm{a}$ & $13,1 \mathrm{a}$ & $91 \mathrm{ab}$ \\
\hline & 2 & 6,3 & $100 \mathrm{a}$ & $41,6 \mathrm{a}$ & $100 \mathrm{a}$ & $32,0 \mathrm{a}$ & $11,8 \mathrm{a}$ & $95 \mathrm{a}$ \\
\hline & 3 & 6,5 & $96 \mathrm{~b}$ & $34,5 \mathrm{c}$ & $97 \mathrm{a}$ & $25,6 \mathrm{~b}$ & $13,2 \mathrm{a}$ & $86 \mathrm{~b}$ \\
\hline & c.v. $(\%)$ & - & 1,3 & 3,3 & 2,5 & 4,4 & 7,1 & 4,1 \\
\hline \multirow{4}{*}{ Jóia } & A & 6,7 & $99 a$ & $35,4 \mathrm{a}$ & $100 \mathrm{a}$ & $32,1 \mathrm{a}$ & $14,7 \mathrm{a}$ & $98 \mathrm{a}$ \\
\hline & B & 6,8 & $97 \mathrm{a}$ & $32,1 \mathrm{~b}$ & $98 a b$ & $29,6 \mathrm{~b}$ & $14,5 \mathrm{a}$ & $95 \mathrm{ab}$ \\
\hline & $\mathrm{C}$ & 6,7 & $98 \mathrm{a}$ & $31,9 \mathrm{~b}$ & $97 \mathrm{~b}$ & $23,4 \mathrm{c}$ & $13,9 \mathrm{a}$ & $90 \mathrm{~b}$ \\
\hline & c.v. $(\%)$ & - & 2,2 & 2,6 & 1,5 & 4,3 & 4,1 & 3,6 \\
\hline
\end{tabular}

Letras minúsculas: comparação das médias em cada coluna (Tukey a 5\% de probabilidade), separadamente para cada cultivar. 
O teor de água inicial das sementes variou em 0,5 ponto porcentual $(6,3 \%$ a $6,8 \%)$, estando dentro do limite tolerável para a obtenção de resultados consistentes (Marcos Filho, 2005).

A utilização de lotes com diferentes níveis de vigor é importante para o estudo da relação entre o potencial fisiológico e a resposta das sementes ao condicionamento. Considerandose que a técnica tem por objetivo uniformizar o comportamento de indivíduos componentes de uma população (Marcos Filho, 2005), lotes compostos por sementes em avançado estádio de deterioração podem não responder adequadamente ao condicionamento fisiológico; da mesma forma, geralmente, não há como beneficiar lotes altamente vigorosos.

\section{Avaliação dos procedimentos para o condicionamento fisiológico}

$\mathrm{Na}$ Tabela 2 estão os teores de água antes e após o condicionamento fisiológico a $25^{\circ} \mathrm{C}$. Verifica-se que os teores finais obtidos estavam muito próximos aos esperados, ou seja, 29\% para os lotes do cv Safira e 32\% para os lotes do cv Jóia, contribuindo para a obtenção de resultados precisos.

TABELA2. Teores de água de lotes de sementes do cv Safira e do cv Jóia antes (inicial)e após (final) o osmocondicionamento em papel embebido em solução de PEG -0,1MPa e PEG -0,2MPa e tempo médio (T médio) para as sementes atingirem o teor de água desejado.

\begin{tabular}{|c|c|c|c|c|c|c|c|c|c|}
\hline \multirow{3}{*}{ Tratamentos } & \multicolumn{9}{|c|}{ Híbrido "Safira" } \\
\hline & \multicolumn{3}{|c|}{ Lote 1} & \multicolumn{3}{|c|}{ Lote 2} & \multicolumn{3}{|c|}{ Lote 3} \\
\hline & inicial & final & T médio & inicial & final & T médio & inicial & final & T médio \\
\hline PEG -0,1MPa & 6,2 & 29,1 & $10 \mathrm{~h}$ & 6,3 & 28,8 & $10 \mathrm{~h}$ & 6,3 & 28,5 & $10 \mathrm{~h}$ \\
\hline PEG -0,2MPa & 6,2 & 29,0 & $12 \mathrm{~h} 30 \mathrm{~min}$ & 6,3 & 28,8 & $11 \mathrm{~h} 30 \mathrm{~min}$ & 6,3 & 28,7 & $12 \mathrm{~h} 30 \mathrm{~min}$ \\
\hline Testemunha & & 6 & & & 6, & & & 6,8 & \\
\hline \multirow{3}{*}{ Tratamentos } & \multicolumn{9}{|c|}{ Híbrido "Jóia" } \\
\hline & \multicolumn{3}{|c|}{ Lote A } & \multicolumn{3}{|c|}{ Lote B } & \multicolumn{3}{|c|}{ Lote C } \\
\hline & inicial & final & $\mathrm{T}$ médio & inicial & final & T médio & inicial & final & $\mathrm{T}$ médio \\
\hline PEG -0,1MPa & 7,2 & 32,5 & $16 \mathrm{~h}$ & 7,3 & 32,2 & $16 \mathrm{~h} 10 \mathrm{~min}$ & 7,1 & 32,0 & $16 \mathrm{~h} 30 \mathrm{~min}$ \\
\hline PEG -0,2MPa & 7,2 & 32,0 & $17 \mathrm{~h} 15 \mathrm{~min}$ & 7,3 & 31,6 & $19 \mathrm{~h} 45 \mathrm{~min}$ & 7,1 & 31,8 & $20 \mathrm{~h}$ \\
\hline Testemunha & \multicolumn{3}{|c|}{7,2} & \multicolumn{3}{|c|}{7,3} & \multicolumn{3}{|c|}{7,1} \\
\hline
\end{tabular}

Na Tabela 3 estão os dados referentes ao teste de germinação de sementes condicionadas e a testemunha a $15{ }^{\circ} \mathrm{C}$. Nessa temperatura, o condicionamento fisiológico favoreceu tanto a porcentagem quanto a velocidade de germinação, em relação à testemunha.

Devido à temperatura de $15{ }^{\circ} \mathrm{C}$ ser drástica para a espécie, as sementes não haviam germinado até a data da primeira contagem do teste; no entanto, o condicionamento fisiológico promoveu efeitos positivos, proporcionando uma elevação na porcentagem e velocidade de germinação em todos os lotes testados. Um dos aspectos vantajosos do condicionamento fisiológico, citado na literatura, é seu efeito benéfico em condições de estresse (Guedes e Cantliffe, 1980; Trigo e Trigo, 1999; Nascimento e Lima, 2008; Pereira et al., 2009).

Para os lotes 1 e 2, o osmocondicionamento com embebição do papel toalha em solução de PEG -0,1MPa promoveu resultados de porcentagem e velocidade de germinação a $15{ }^{\circ} \mathrm{C}$ mais elevados que a testemunha e que o osmocondicionamento com PEG -0,2MPa. No entanto, o osmocondicionamento com solução de PEG -0,2 MPa também promoveu resultados benéficos e superiores a testemunha. Para o lote 3 , o melhor resultado foi verificado com o uso de PEG -0,2 MPa. Para os lotes do cultivar Jóia, o uso de PEG -0,2 MPa favoreceu a porcentagem e a velocidade de germinação. Potenciais osmóticos mais baixos determinam uma embebição mais lenta, o que permite maior período de tempo para que os tecidos do embrião se reorganizem e levam a um melhor desempenho das sementes. Por outro lado, potenciais mais elevados podem promover uma embebição lenta, porém insuficiente para permitir a reorganização das membranas celulares a ponto de determinar um melhor desempenho das sementes, como ocorreu com os lotes do cv. Safira osmocondicionados em papel embebido em solução de PEG -0,1 MPa. 
TABELA 3. Resultados das avaliações de primeira contagem do teste de germinação (1 ${ }^{\mathrm{a}} \mathrm{C}$.), porcentagem (Germ.) e velocidade de germinação (V.G.) a $15^{\circ} \mathrm{C}$, de sementes de pepino cv. Safira (lotes 1, 2 e 3) e cv. Jóia (lotes A, B e C) submetidas ou não (testemunha) ao osmocondicionamento a $25^{\circ} \mathrm{C}$ com embebição de papel em solução de PEG -0,1MPa e -0,2MPa.

\begin{tabular}{|c|c|c|c|c|c|c|c|c|c|}
\hline \multirow[b]{2}{*}{ Tratamentos } & \multicolumn{3}{|c|}{ Lote 1} & \multicolumn{3}{|c|}{ Lote 2} & \multicolumn{3}{|c|}{ Lote 3} \\
\hline & $\begin{array}{c}1^{\mathrm{a}} \mathrm{C} . \\
\ldots . . \% \ldots\end{array}$ & $\begin{array}{c}\text { Germ. } \\
\ldots . . . \% \ldots\end{array}$ & $\begin{array}{l}\text { V.G. } \\
\text { índice }\end{array}$ & $\begin{array}{c}1^{\mathrm{a}} \mathrm{C} . \\
\ldots . . \%\end{array}$ & $\begin{array}{c}\text { Germ. } \\
\ldots . . \% \ldots\end{array}$ & $\begin{array}{l}\text { V.G. } \\
\text { índice }\end{array}$ & $\begin{array}{c}1^{\mathrm{a}} \mathrm{C} . \\
\ldots . \% \% \ldots\end{array}$ & $\begin{array}{c}\text { Germ. } \\
\ldots . . \% \ldots . . .\end{array}$ & $\begin{array}{l}\text { V.G. } \\
\text { índice }\end{array}$ \\
\hline Testemunha & 0 & $33 \mathrm{c}$ & $4,3 \mathrm{c}$ & 0 & $41 \mathrm{c}$ & $5,2 \mathrm{~b}$ & 0 & $22 \mathrm{c}$ & $2,6 \mathrm{c}$ \\
\hline PEG -0,1MPa & 0 & $74 \mathrm{a}$ & $10,4 \mathrm{a}$ & 0 & $92 \mathrm{a}$ & $13,5 \mathrm{a}$ & 0 & $55 \mathrm{~b}$ & $7,5 \mathrm{~b}$ \\
\hline PEG -0,2MPa & 0 & $58 \mathrm{~b}$ & $8,2 \mathrm{~b}$ & 0 & $89 \mathrm{~b}$ & $7,2 \mathrm{~b}$ & 0 & $72 \mathrm{a}$ & $10,2 \mathrm{a}$ \\
\hline c.v. $(\%)$ & 0 & 13,4 & 13,5 & 0 & 9,2 & 12,7 & 0 & 9,3 & 10,6 \\
\hline Tratamentos & & Lote A & & & Lote B & & & ote C & \\
\hline Testemunha & 0 & $2 c$ & $0,1 \mathrm{c}$ & 0 & $0 \mathrm{c}$ & $0 \mathrm{c}$ & 0 & $0 \mathrm{c}$ & $0 \mathrm{c}$ \\
\hline PEG -0,1MPa & 0 & $73 \mathrm{~b}$ & $9,9 \mathrm{~b}$ & 0 & $75 \mathrm{~b}$ & $10,3 \mathrm{~b}$ & 0 & $60 \mathrm{~b}$ & $7,7 \mathrm{~b}$ \\
\hline PEG -0,2MPa & 0 & $97 \mathrm{a}$ & $15,2 \mathrm{a}$ & 0 & $90 \mathrm{a}$ & $13,6 \mathrm{a}$ & 0 & $72 \mathrm{a}$ & $10,8 \mathrm{a}$ \\
\hline c.v. $(\%)$ & 0 & 5,5 & 5,3 & 0 & 4,3 & 5,3 & 0 & 10,1 & 13,4 \\
\hline
\end{tabular}

Letras minúsculas: comparações entre médias na mesma coluna (Tukey, $\mathrm{p} \leq 0,05$ ).

Entretanto, quando as sementes foram colocadas para germinar a $20{ }^{\circ} \mathrm{C}$ (Tabela 4), o aumento da temperatura em $5{ }^{\circ} \mathrm{C}$ (de 15 para $20{ }^{\circ} \mathrm{C}$ ) permitiu que as sementes germinassem mais rapidamente. Assim, pode-se observar que para os lotes do cv. Safira, o osmocondicionamento com embebição do papel em solução de PEG -0,2 MPa, promoveu incremento na velocidade de germinação.

TABELA 4. Resultados das avaliações de primeira contagem do teste de germinação (1 ${ }^{\mathrm{a}} \mathrm{C}$.), porcentagem (Germ.) e velocidade de germinação (V.G.) a $20^{\circ} \mathrm{C}$, de sementes de pepino cv Safira (lotes 1, 2 e 3) e cv Jóia (lotes A, B e C) submetidas ou não (testemunha) ao osmocondicionamento a $25^{\circ} \mathrm{C}$ com embebição de papel em solução de PEG -0,1MPa e -0,2MPa.

\begin{tabular}{|c|c|c|c|c|c|c|c|c|c|}
\hline \multirow[b]{2}{*}{ Tratamentos } & \multicolumn{3}{|c|}{ Lote 1} & \multicolumn{3}{|c|}{ Lote 2} & \multicolumn{3}{|c|}{ Lote 3} \\
\hline & $\begin{array}{l}1^{\mathrm{a}} \mathrm{C} . \\
\ldots \% \ldots\end{array}$ & $\begin{array}{c}\text { Germ. } \\
\ldots . . \% \ldots . . .\end{array}$ & $\begin{array}{l}\text { V.G. } \\
\text { índice }\end{array}$ & $\begin{array}{c}1^{\mathrm{a}} \mathrm{C} . \\
\ldots . . \% \ldots . .\end{array}$ & $\begin{array}{c}\text { Germ. } \\
\ldots . . \% \ldots . . .\end{array}$ & $\begin{array}{l}\text { V.G. } \\
\text { índice }\end{array}$ & $\begin{array}{c}1^{\mathrm{a}} \mathrm{C} . \\
\ldots . . \% \ldots\end{array}$ & $\begin{array}{c}\text { Germ. } \\
\ldots . . \% \ldots . . .\end{array}$ & $\begin{array}{l}\text { V.G. } \\
\text { índice }\end{array}$ \\
\hline Testemunha & $49 \mathrm{~b}$ & $100 \mathrm{a}$ & $22,3 \mathrm{~b}$ & $89 \mathrm{a}$ & $99 \mathrm{a}$ & $24,2 \mathrm{~b}$ & $36 \mathrm{~b}$ & $98 \mathrm{a}$ & $21,3 \mathrm{~b}$ \\
\hline PEG -0,1MPa & $36 \mathrm{c}$ & $96 \mathrm{~b}$ & $20,9 \mathrm{c}$ & $39 \mathrm{~b}$ & 98 a & $21,5 \mathrm{c}$ & $17 \mathrm{c}$ & 96 a & $20,0 \mathrm{c}$ \\
\hline PEG -0,2MPa & $96 \mathrm{a}$ & $99 a$ & $28,5 \mathrm{a}$ & 99 a & $100 \mathrm{a}$ & $32,3 \mathrm{a}$ & $89 a$ & $96 \mathrm{a}$ & $26,7 \mathrm{a}$ \\
\hline c.v. $(\%)$ & 10,9 & 1,7 & 2,3 & 8,4 & 1,1 & 2,1 & 10,1 & 3,0 & 2,7 \\
\hline Tratamentos & \multicolumn{3}{|c|}{ Lote A } & \multicolumn{3}{|c|}{ Lote B } & \multicolumn{3}{|c|}{ Lote $\mathrm{C}$} \\
\hline Testemunha & $56 \mathrm{~b}$ & 99 a & $22,4 \mathrm{~b}$ & $12 b$ & $95 \mathrm{a}$ & $19,4 \mathrm{~b}$ & $0 \mathrm{~b}$ & $87 \mathrm{a}$ & $16,8 \mathrm{~b}$ \\
\hline PEG -0,1MPa & $97 \mathrm{a}$ & 99 a & $32,2 \mathrm{a}$ & $94 \mathrm{a}$ & $97 \mathrm{a}$ & $28,4 \mathrm{a}$ & $76 \mathrm{a}$ & $92 \mathrm{a}$ & $22,5 \mathrm{a}$ \\
\hline PEG -0,2MPa & $99 \mathrm{a}$ & 99 a & $31,6 \mathrm{a}$ & $91 \mathrm{a}$ & $94 \mathrm{a}$ & $27,4 \mathrm{a}$ & $77 \mathrm{a}$ & $94 \mathrm{a}$ & $23,3 \mathrm{a}$ \\
\hline c.v. $(\%)$ & 8,1 & 1,4 & 1,9 & 7,0 & 4,5 & 4,7 & 15,1 & 5,9 & 7,3 \\
\hline
\end{tabular}

Letras minúsculas: comparações entre médias na mesma coluna (Tukey, $\mathrm{p} \leq 0,05$ ).

Para os lotes do cultivar Jóia (lotes A, B e C), o condicionamento não alterou significativamente a porcentagem de germinação a $20^{\circ} \mathrm{C}$; no entanto, verificou- se efeito positivo na primeira contagem do teste de germinação e na velocidade de germinação, para ambos os procedimentos de condicionamento fisiológico testados. 
Nesse cultivar, o efeito mais expressivo do condicionamento fisiológico foi observado nos lotes de menor vigor (lotes $\mathrm{B}$ e C), onde o incremento na porcentagem de germinação na primeira contagem e na velocidade de germinação foram, proporcionalmente, os mais evidentes dentre todos os lotes testados. Rao et al. (1987) e Nascimento e Aragão (2004) também verificaram, respectivamente, em sementes de brássicas e de melão, efeitos benéficos do condicionamento fisiológico em sementes de lotes de vigor inferior, quando avaliadas em temperaturas subótimas.

Quando a embebição das sementes ocorre em temperaturas subótimas, como 15 e $20{ }^{\circ} \mathrm{C}$ no caso do pepino (Filgueira, 2000; Canizares, 1998), podem ocorrer injúrias por estresse térmico, provavelmenterelacionadoàsdanificaçõesaosistemademembranas, que prejudicam a germinação das sementes e o estabelecimento das plântulas. Assim, a possibilidade da reestruturação do sistema de membranas durante o condicionamento fisiológico determina a manutenção da permeabilidade seletiva, de forma que as membranas organizadas não permitem a entrada rápida de água nem a liberação excessiva de exsudados quando a embebição é retomada (Marcos Filho, 2005).

Com a aplicação do condicionamento fisiológico em sementes de pimentão, Roveri-José et al. (2000) e Posse et al. (2001) obtiveram incrementos expressivos na germinação das sementes em temperaturas inferiores à ideal para a espécie, semelhante ao observado em sementes de berinjela (Nascimento e Lima, 2008), de melão (Nascimento, 1999; Nascimento e Aragão, 2004) e de cenoura (Pereira et al.,
2009). Para sementes de canola (Zengh et al., 1994) e de couve-flor (Fujikura et al., 1993) o condicionamento fisiológico proporcionou maior velocidade e uniformidade de germinação em condições de baixa temperatura. Da mesma forma, em sementes de pepino, Thanos e Georghiou (1988) verificaram que o condicionamento fisiológico promoveu aumento considerável na velocidade de germinação a 15 e 20 ${ }^{\circ} \mathrm{C}$, visto que a germinação das sementes não condicionadas foi extremamente afetada pelas temperaturas subótimas. Staub et al. (1986) observaram que o condicionamento fisiológico em sementes de pepino poderia promover efeitos positivos de forma mais acentuada em situação de temperatura subótima do que sob temperatura ideal $\left(25^{\circ} \mathrm{C}\right)$.

Assim, corroborando com os resultados desses autores, o efeito benéfico do condicionamento fisiológico sobre a porcentagem e/ou velocidade de germinação de sementes em temperaturas subótimas (Khan, 1992; Nascimento, 2005; Nascimento e Lima, 2008; Pereira et al., 2009) foi confirmado no presente trabalho para sementes de pepino.

Na Tabela 5, pode-se observar os resultados da germinação a $25{ }^{\circ} \mathrm{C}$. Para os lotes A e C, verificou-se efeito positivo na velocidade de germinação após o osmocondicionamento das sementes com solução de PEG -0,2 MPa, enquanto para o lote $\mathrm{B}$, não houve diferença entre o uso de solução de PEG -0,1 MPa ou -0,2 MPa, sendo que ambos favoreceram a velocidade de germinação. Para o lote 3, o maior índice de velocidade de germinação foi obtido com o uso de solução de PEG - 0,1 $\mathrm{MPa}$, porém não diferindo da testemunha.

TABELA 5. Resultados das avaliações de primeira contagem do teste de germinação (1 ${ }^{\mathrm{a}} \mathrm{C}$.), porcentagem (Germ.) e velocidade de germinação (V.G.) a $25^{\circ} \mathrm{C}$, de sementes de pepino cv Safira (lotes 1,2 e 3) e cv Jóia (lotes A, B e C) submetidas ou não (testemunha) ao osmocondicionamento a $25^{\circ} \mathrm{C}$ com embebição de papel em solução de PEG -0,1 MPa e -0,2 MPa.

\begin{tabular}{|c|c|c|c|c|c|c|c|c|c|}
\hline \multirow[b]{2}{*}{ Tratamentos } & \multicolumn{3}{|c|}{ Lote 1} & \multicolumn{3}{|c|}{ Lote 2} & \multicolumn{3}{|c|}{ Lote 3} \\
\hline & $\begin{array}{c}1^{\mathrm{a}} \mathrm{C} . \\
\ldots . . . \% \ldots\end{array}$ & $\begin{array}{c}\text { Germ. } \\
\ldots . . . \% \ldots\end{array}$ & $\begin{array}{l}\text { V.G. } \\
\text { índice }\end{array}$ & $\begin{array}{c}1^{\mathrm{a}} \mathrm{C} . \\
\ldots . . \% \ldots\end{array}$ & $\begin{array}{c}\text { Germ. } \\
\ldots . . . \% . . .\end{array}$ & $\begin{array}{l}\text { V.G. } \\
\text { índice }\end{array}$ & $\begin{array}{c}1^{\mathrm{a}} \mathrm{C} . \\
\ldots . . \%\end{array}$ & $\begin{array}{l}\text { Germ. } \\
\ldots . . \% \ldots\end{array}$ & $\begin{array}{l}\text { V.G. } \\
\text { índice }\end{array}$ \\
\hline Testemunha & $100 \mathrm{a}$ & $100 \mathrm{a}$ & $44,8 \mathrm{a}$ & $100 \mathrm{a}$ & $100 \mathrm{a}$ & $49,4 \mathrm{a}$ & 99 a & 99 a & $43,8 \mathrm{ab}$ \\
\hline PEG -0,1MPa & $100 \mathrm{a}$ & $100 \mathrm{a}$ & $44,4 \mathrm{a}$ & $99 \mathrm{a}$ & $100 \mathrm{a}$ & $49,1 \mathrm{a}$ & $98 a b$ & $98 \mathrm{a}$ & $45,2 \mathrm{a}$ \\
\hline PEG -0,2MPa & $99 \mathrm{a}$ & 99 a & 43,6 a & $100 \mathrm{a}$ & $100 \mathrm{a}$ & $49,4 \mathrm{a}$ & $95 \mathrm{~b}$ & 99 a & $41,4 \mathrm{~b}$ \\
\hline c.v. $(\%)$ & 1,1 & 1,0 & 2,0 & 1,0 & 1,0 & 1,0 & 1,7 & 1,7 & 4,1 \\
\hline Tratamentos & \multicolumn{3}{|c|}{ Lote A } & \multicolumn{3}{|c|}{ Lote B } & \multicolumn{3}{|c|}{ Lote C } \\
\hline Testemunha & $100 \mathrm{a}$ & $100 \mathrm{a}$ & $47,5 \mathrm{~b}$ & $93 \mathrm{a}$ & $96 a$ & $35,2 \mathrm{~b}$ & $94 \mathrm{a}$ & $95 \mathrm{a}$ & $30,6 \mathrm{c}$ \\
\hline PEG -0,1MPa & $98 \mathrm{a}$ & 99 a & $48,2 \mathrm{ab}$ & $96 \mathrm{a}$ & $97 \mathrm{a}$ & $46,1 \mathrm{a}$ & $91 \mathrm{a}$ & $92 \mathrm{a}$ & $35,6 \mathrm{~b}$ \\
\hline PEG -0,2MPa & $100 \mathrm{a}$ & $100 \mathrm{a}$ & $49,1 \mathrm{a}$ & $96 a$ & $96 a$ & $46,6 \mathrm{a}$ & $92 \mathrm{a}$ & $92 \mathrm{a}$ & $44,6 \mathrm{a}$ \\
\hline c.v. $(\%)$ & 1,1 & 1,0 & 1,3 & 2,3 & 2,1 & 2,1 & 5,8 & 5,3 & 6,8 \\
\hline
\end{tabular}

Letras minúsculas: comparações entre médias na mesma coluna (Tukey, $\mathrm{p} \leq 0,05$ ). 
Os efeitos do condicionamento fisiológico foram mais evidentes nos lotes de menor vigor (lotes 3 e C), corroborando os resultados obtidos por Trigo et al. (1999) em sementes de cebola, em que a velocidade de germinação foi favorecida pelo tratamento em sementes de baixo vigor; e com os resultados de Bradford et al. (1990) para sementes de pimentão e Burgass e Powell (1984) em sementes de brássicas, onde os lotes de menor vigor tiveram os maiores incrementos nos parâmetros avaliados com a aplicação da técnica. Szafirowska et al. (1981) observaram que as vantagens do condicionamento fisiológico podem ser mínimas em lotes de alto vigor.

Em sementes de melão colocadas para germinar a 25 ${ }^{\circ} \mathrm{C}$, temperatura ideal para a espécie, Nascimento (1999) observou que o condicionamento fisiológico foi benéfico à velocidade de germinação, semelhante ao verificado em sementes de alface (Eira, 1988).

Na Tabela 6 estão os resultados obtidos utilizandose a temperatura de $30{ }^{\circ} \mathrm{C}$ para a condução do teste de germinação das sementes tratadas. De um modo geral, não se verificou efeito do condicionamento fisiológico, independentemente do procedimento utilizado. Apenas no lote $\mathrm{B}$, o condicionamento com PEG - $0,1 \mathrm{MPa}$, favoreceu o índice de velocidade de germinação. Resultado semelhante foi obtido quando o teste de germinação foi conduzido a $35{ }^{\circ} \mathrm{C}$ (Tabela 7), onde os resultados obtidos com o condicionamento não diferiram da testemunha para os lotes do cv. Safira e para o lote A do cv. Jóia. Nos lotes B e C o condicionamento fisiológico favoreceu a velocidade de germinação em relação à testemunha.

TABELA 6. Resultados das avaliações de primeira contagem do teste de germinação (1ª C.), porcentagem (Germ.) e velocidade de germinação (V.G.) a $30^{\circ} \mathrm{C}$, de sementes de pepino cv Safira (lotes 1, 2 e 3) e cv Jóia (lotes A, B e C) submetidas ou não (testemunha) ao osmocondicionamento a $25{ }^{\circ} \mathrm{C}$ com embebição de papel em solução de PEG -0,1 MPa e -0,2 MPa.

\begin{tabular}{|c|c|c|c|c|c|c|c|c|c|}
\hline \multirow[b]{2}{*}{ Tratamentos } & \multicolumn{3}{|c|}{ Lote 1} & \multicolumn{3}{|c|}{ Lote 2} & \multicolumn{3}{|c|}{ Lote 3} \\
\hline & $\begin{array}{c}1^{\mathrm{a}} \mathrm{C} \\
\ldots . \% \ldots\end{array}$ & $\begin{array}{l}\text { Germ. } \\
\ldots . \% \ldots\end{array}$ & $\begin{array}{l}\text { V.G. } \\
\text { índice }\end{array}$ & $\begin{array}{c}1^{\mathrm{a}} \mathrm{C} . \\
\ldots \% \ldots\end{array}$ & $\begin{array}{l}\text { Germ. } \\
\ldots . . \% \ldots . . .\end{array}$ & $\begin{array}{l}\text { V.G. } \\
\text { índice }\end{array}$ & $\begin{array}{c}1^{\mathrm{a}} \mathrm{C} . \\
\ldots . . \% \ldots\end{array}$ & $\begin{array}{c}\text { Germ. } \\
\ldots . . \% \ldots\end{array}$ & $\begin{array}{l}\text { V.G. } \\
\text { índice }\end{array}$ \\
\hline Testemunha & $98 \mathrm{a}$ & $98 \mathrm{a}$ & $48,1 \mathrm{a}$ & $100 \mathrm{a}$ & $100 \mathrm{a}$ & $49,4 \mathrm{a}$ & $95 \mathrm{a}$ & $95 \mathrm{a}$ & $46,6 \mathrm{a}$ \\
\hline PEG -0,1MPa & $99 \mathrm{a}$ & $100 \mathrm{a}$ & $49,6 \mathrm{a}$ & $100 \mathrm{a}$ & $100 \mathrm{a}$ & $49,7 \mathrm{a}$ & $98 \mathrm{a}$ & $98 \mathrm{a}$ & $48,2 \mathrm{a}$ \\
\hline PEG -0,2MPa & $98 \mathrm{a}$ & $99 a$ & $48,9 \mathrm{a}$ & $99 \mathrm{a}$ & $99 a$ & $49,0 \mathrm{a}$ & $97 \mathrm{a}$ & $97 \mathrm{a}$ & $48,1 \mathrm{a}$ \\
\hline c.v. $(\%)$ & 2,0 & 1,6 & 1,9 & 1,9 & 1,9 & 1,7 & 2.5 & 2,5 & 2,3 \\
\hline Tratamentos & \multicolumn{3}{|c|}{ Lote A } & \multicolumn{3}{|c|}{ Lote B } & \multicolumn{3}{|c|}{ Lote C } \\
\hline Testemunha & 99 a & $99 a$ & $49,4 \mathrm{a}$ & $94 \mathrm{a}$ & $94 \mathrm{a}$ & $46,3 \mathrm{~b}$ & $93 \mathrm{a}$ & $93 \mathrm{a}$ & $44,9 \mathrm{a}$ \\
\hline PEG -0,1MPa & 99 a & $99 a$ & $49,5 \mathrm{a}$ & $98 \mathrm{a}$ & $98 \mathrm{a}$ & $49,0 \mathrm{a}$ & $93 \mathrm{a}$ & $93 \mathrm{a}$ & $46,2 \mathrm{a}$ \\
\hline PEG -0,2MPa & $100 \mathrm{a}$ & $100 \mathrm{a}$ & $49,7 \mathrm{a}$ & $98 \mathrm{a}$ & $98 \mathrm{a}$ & $48,7 \mathrm{ab}$ & $92 \mathrm{a}$ & $93 a$ & $46,1 \mathrm{a}$ \\
\hline c.v. $(\%)$ & 1,0 & 1,0 & 1,2 & 2,5 & 2,5 & 2,7 & 4,1 & 3,8 & 4,3 \\
\hline
\end{tabular}

Letras minúsculas: comparações entre médias na mesma coluna (Tukey, $\mathrm{p} \leq 0,05$ ).

Pela análise dos resultados do condicionamento fisiológico obtidos em diferentes temperaturas, o procedimento utilizando solução de PEG $-0,2 \mathrm{MPa}$ mostrou-se o mais promissor. Salienta-se que no potencial hídrico final do sistema em estudo existe a contribuição do potencial matricial do papel toalha utilizado.

Segundo Nascimento (2000), a temperatura máxima para a germinação de sementes de pepino é $41^{\circ} \mathrm{C}$, enquanto a faixa ideal encontra-se entre 25 e $30{ }^{\circ} \mathrm{C}$ (Canizares, 1998). Assim, temperaturas superiores à ótima, como $35^{\circ} \mathrm{C}$, provocam aumento na velocidade de germinação (Nascimento, 2000), enquanto na faixa ideal ocorre a máxima porcentagem de germinação no menor período de tempo. Desta forma, o condicionamento fisiológico não teve efeito sobre as sementes quando colocadas para germinar em temperaturas altas (Tabelas 6 e 7). Assim, como a porcentagem e a velocidade de germinação das testemunhas foram estimuladas principalmente pela alta temperatura durante o teste, os efeitos positivos verificados não foram considerados relevantes. 
TABELA 7. Resultados das avaliações de primeira contagem do teste de germinação (1 ${ }^{\mathrm{a}} \mathrm{C}$.), porcentagem (Germ.) e velocidade de germinação (V.G.) a $35^{\circ} \mathrm{C}$, de sementes de pepino cv. Safira (lotes 1, 2 e 3) e cv. Jóia (lotes A, B e C) submetidas ou não (testemunha) ao osmocondicionamento a $25^{\circ} \mathrm{C}$ com embebição de papel em solução de PEG -0,1 MPa e -0,2 MPa.

\begin{tabular}{|c|c|c|c|c|c|c|c|c|c|}
\hline \multirow[b]{2}{*}{ Tratamentos } & \multicolumn{3}{|c|}{ Lote 1} & \multicolumn{3}{|c|}{ Lote 2} & \multicolumn{3}{|c|}{ Lote 3} \\
\hline & $\begin{array}{c}1^{\mathrm{a}} \mathrm{C} . \\
\ldots . . \% \ldots . .\end{array}$ & $\begin{array}{c}\text { Germ. } \\
\ldots . . \% \ldots . . .\end{array}$ & $\begin{array}{l}\text { V.G. } \\
\text { índice }\end{array}$ & $\begin{array}{c}1^{\mathrm{a}} \mathrm{C} . \\
\ldots . . \% \ldots . .\end{array}$ & $\begin{array}{c}\text { Germ. } \\
\ldots . . \% \ldots . . .\end{array}$ & $\begin{array}{l}\text { V.G. } \\
\text { índice }\end{array}$ & $\begin{array}{c}1^{\mathrm{a}} \mathrm{C} . \\
\ldots . . \% \ldots\end{array}$ & $\begin{array}{c}\text { Germ. } \\
\ldots . . \% \ldots . . .\end{array}$ & $\begin{array}{l}\text { V.G. } \\
\text { índice }\end{array}$ \\
\hline Testemunha & $98 \mathrm{~b}$ & $98 \mathrm{ab}$ & $48,6 \mathrm{a}$ & $100 \mathrm{a}$ & $100 \mathrm{a}$ & $49,6 \mathrm{a}$ & $95 \mathrm{a}$ & $95 \mathrm{a}$ & $46,0 \mathrm{a}$ \\
\hline PEG -0,1MPa & $97 \mathrm{~b}$ & $97 \mathrm{~b}$ & $46,7 \mathrm{a}$ & $100 \mathrm{a}$ & $100 \mathrm{a}$ & $49,6 \mathrm{a}$ & $86 \mathrm{a}$ & $86 \mathrm{a}$ & $41,0 \mathrm{a}$ \\
\hline PEG -0,2MPa & $100 \mathrm{a}$ & $100 \mathrm{a}$ & $46,7 \mathrm{a}$ & $100 \mathrm{a}$ & $100 \mathrm{a}$ & $49,7 \mathrm{a}$ & $96 a$ & $96 a$ & $44,0 \mathrm{a}$ \\
\hline c.v. $(\%)$ & 1,3 & 1.3 & 2,9 & 1,0 & 1,0 & 1,1 & 6,6 & 6,6 & 6,2 \\
\hline Tratamentos & \multicolumn{3}{|c|}{ Lote A } & \multicolumn{3}{|c|}{ Lote B } & \multicolumn{3}{|c|}{ Lote C } \\
\hline Testemunha & $98 \mathrm{a}$ & $99 a$ & $47,8 \mathrm{a}$ & $97 \mathrm{a}$ & $97 \mathrm{a}$ & $41,3 \mathrm{~b}$ & $90 \mathrm{a}$ & $91 \mathrm{a}$ & $35,3 \mathrm{~b}$ \\
\hline PEG -0,1MPa & $98 \mathrm{a}$ & $98 \mathrm{a}$ & $48,8 \mathrm{a}$ & $94 \mathrm{a}$ & $94 \mathrm{a}$ & $45,6 \mathrm{a}$ & $89 a$ & 89 a & $39,1 \mathrm{ab}$ \\
\hline PEG -0,2MPa & $98 \mathrm{a}$ & $98 \mathrm{a}$ & $47,3 \mathrm{a}$ & $98 \mathrm{a}$ & $98 \mathrm{a}$ & $46,8 \mathrm{a}$ & $91 \mathrm{a}$ & $91 \mathrm{a}$ & $43,1 \mathrm{a}$ \\
\hline c.v. $(\%)$ & 2,9 & 3,0 & 3,0 & 3,6 & 3,6 & 3,8 & 7,3 & 7,4 & 7,6 \\
\hline
\end{tabular}

Letras minúsculas: comparações entre médias na mesma coluna (Tukey, $\mathrm{p} \leq 0,05$ ).

\section{CONCLUSÃO}

O osmocondicionamento a $25^{\circ} \mathrm{C}$, com embebição do papel em solução de PEG -0,2 MPa, é benéfico ao desempenho de sementes de pepino e aumenta a velocidade de germinação para a maioria dos lotes e temperaturas testados.

\section{AGRADECIMENTOS}

Às empresas Sakata Seed Sudamerica e Seminis Vegetable Seeds pela cessão das sementes.

À FAPESP, pela concessão da bolsa de estudo e suporte financeiro ao projeto.

\section{REFERÊNCIAS}

ANDREOLI, C.; KHAN, A. Matriconditioning integrated with gibberellic acid to hasten seed germination and improve stand establishment of pepper and tomato. Pesquisa Agropecuária Brasileira, v.34, n.10, p.1953-1958, 1999.

BRADFORD, K.J.; STEINER, J.J.; TRAWATHA, S.E. Seed priming influence on germination and emergence of pepper seed lots. Crop Science, v.30, n 3, p.718-721, 1990.

BRASIL. Ministério da Agricultura e Reforma Agrária. Secretaria Nacional de Defesa Agropecuária. Departamento Nacional de Produção Vegetal. Regras para Análise de Sementes. Brasília, DF, 1992. 365p.
BURGASS, R.W.; POWELL, A.A. Evidence for repair processes in the invigoration of seeds by hydration. Annals of Botany, v.53, n.5, p.753-757, 1984.

CAÑIZARES, K.A.L. A cultura do pepino. In: GOTO, R; TIVELli, S.W. (Org.). Produção de hortaliças em ambiente protegido: condições subtropicais. Editora UNESP, São Paulo.1998. p.195-223.

CARNEIRO, J.W.P.; BRACCINI, A.L.; GUEDES, T.A.; AMARAL, D.do. Influência do estresse hídrico, térmico e do condicionamento osmótico no desempenho germinativo de sementes de cenoura (Daucus carota L.). Revista Brasileira de Sementes, v.21, n.2, p.208-216, 1999.

CASEIRO, R.F.; BENNETT, M.A.; MARCOS FILHO, J. Comparison of three priming techniques for onion seed differing in initial seed quality. Seed Science and Technology, v.32, n.2, p.365-375, 2004.

DIAS, D.C.F.S.; PAIXÃO, G.P.; SEDIYAMA, M.A.N.; CECON, P.R. Pré-condicionamento de sementes de quiabo: efeitos na qualidade fisiológica e no potencial de armazenamento. Revista Brasileira de Sementes, v.21, n.2, p.224-231, 1999.

EIRA, M.T. Condicionamento osmótico de alface: efeitos sobre a germinação e desempenho sob estresse hídrico, salino e térmico, 1998. 90 f. Dissertação (Mestrado na área de Fitotecnia) - Escola Superior de Agricultura 'Luiz de Queiroz’, Universidade de São Paulo, Piracicaba, 1998. 
FANAN, S.; NOVEMBRE, A.D.da L.C. Condicionamento fisiológico de sementes de berinjela. Bragantia, v.66, n.4, p.675-683, 2007.

FESSEL, S.A.; VIEIRA, R.D.; RODRIGUES, T.de J.D.; FAGIOLI, M. Germinação de sementes de alface submetidas a condicionamento osmótico durante o armazenamento. Scientia Agricola, v.59, n.1, p.73-77, 2002.

FILGUEIRA, F.A.R. Novo manual de olericultura: agrotecnologia moderna na produção e comercialização de hortaliças. Viçosa: UFV, 2000. 402 p.

FUJIKURA, Y.; KRAAK, H.L.; BASRA, A.S.; KARSEN, C.M., Hydropriming, a simple and inexpensive priming method. Seed Science and Technology, v.21, n.3, p.639-642, 1993.

GUEDES, A.C.; CANTLIFFE, D.J. Germination of lettuce seeds at high temperature after seed priming. Journal of the American Society for Horticultural Science, v.105, n.6, p.777-781, 1980.

HEYDECKER, W.; HIGGINS, J.; GULLIVER, R.L. Accelerated germination by osmotic seed treatment. Nature, v.246, n.5427, p.42-44, 1973.

JETT, L.W.; WELBAUM, G.E.; MORSE, R.D. Effects of matric and osmotic priming treatments on broccoli seed germination. Journal of American Society for Horticultural Science, v.121, n.3, p.423-429, 1996.

KHAN, A.A. Preplant physiological seed condictioning. Horticultural Reviews, v.13, p.131-181, 1992.

KNYPL, J.S.; KHAN, A.A. Osmoconditioning of soybean seeds to improve performance at suboptimal temperature. Agronomy Journal, v.73, p.112-116, 1981.

MAGUIRE, J.D. Speed of germination-aid in relation evaluation for seedling emergence vigor. Crop Science, v.2, n.2, p.176-177, 1962.

MARCOS FILHO, J. Fisiologia de sementes de plantas cultivadas. Piracicaba: Fealq, 2005, 495p.

MARCOS FILHO, J.; KIKUTI, A.L.P. Condicionamento fisiológico de sementes de couve-flor e desempenho das plantas em campo. Horticultura Brasileira, v.26, n.2, p.165-169, 2008.

NASCIMENTO, W.M. Utilização do condicionamento osmótico de sementes de melão e tomate visando a germinação em baixa temperatura. Informativo ABRATES, v.9, n.1, p.53-55, 1999.

NASCIMENTO, W.M. Temperatura x Germinação. Seed News, v.4, n.4, p.44-45, 2000.
NASCIMENTO, W.M. Muskmelon seed germination and seedling developmente in response to seed priming. Scientia Agricola, v.60, n.1, p.71-75, 2003.

NASCIMENTO, W.M.; ARAGÃO, F.A.S. Muskmelon seed priming in relation to seed vigor. Scientia Agricola, v.61, n.1, p.114-117, 2004.

NASCIMENTO, W.M. Condicionamento osmótico de sementes de hortaliças visando a germinação em condições de temperaturas baixas. Horticultura Brasileira, v.23, n.2, p.211-214, 2005.

NASCIMENTO, W.M.; LIMA, L.B.de. Condicionamento osmótico de sementes de berinjela visando a germinação sob temperaturas baixas. Revista Brasileira de Sementes, v.30, n.2, p.224-227, 2008.

PARERA, C.A.; CANTLIFFE, D.J. Improved germination and modified imbibition of shrunken-2 sweet corn by seed disinfection and solid matrix priming. Journal of American Society for Horticultural Science, v.116, n.6, p.942-945, 1991.

PASSAM, H.C.; KARAVITES, P.I.; PAPANDREOU, A.A.; THANOS, C.A.; GEORGHIOU, K. Osmocondictionig of seeds in relation to growth and fruit yield of aubergine, pepper, cucumber and melon in unheated greenhouse cultivation. Scientia Horticulturae, v.38, n.3-4, p.207-216, 1989.

PEREIRA, M.D.; DIAS, D.C.F.S.; DIAS, L.A.S.; ARAÚJO, E.F. Primed carrot seeds performance under water and temperature stress. Scientia Agricola, v.66, n.2, p.174-179, 2009.

POSSE, S.C.P.; SILVA, R.F.; VIEIRA, H.D.; CATUNDA, P.H.A. Efeitos do condicionamento osmótico e da hidratação na germinação de sementes de pimentão (Capsicum annuum L.) submetidas a baixa temperatura. Revista Brasileira de Sementes, v.23, n.1, p.123-127, 2001.

RAO, S.C.; AKERS, S.W.; AHRING, R.M. Priming brassica seed to improve emergence under different temperatures and soil moisture conditions. Crop Science, v.27, n.4, p.10501053, 1987.

ROSSETTO, C.A.V.; LIMA, T.M.; NAKAGAWA, J. Qualidade fisiológica e potencial de armazenamento de sementes de tomate submetidas ao condicionamento osmótico. Horticultura Brasileira, v.20, n.4, p.630-634, 2002.

ROVERI JOSÉ, S.C.B.R.; VIEIRA， M.G.G.C.; GUIMARÃES, R.M. Efeito da temperatura e do período de condicionamento osmótico na germinação e no vigor de sementes de pimentão. Revista Brasileira de Sementes, v.22, n.2, p.176-184, 2000. 
STAUB, J.E.; NIENHUIS, J.; LOWER, R.L. Effects of seed preconditioning treatments on emergence of cucumber population. HortScience, v.21, n.6, p.1356-1359, 1986.

SZAFIROWSKA, A.; KHAN, A.A.; PECK, N.H. Osmoconditiong of carrot seeds to improve seedling establishment and yield in cold soil. Agronomy Journal, v.73,n. 6 , p.845-848, 1981.

THANOS, C.A.; GEORGHIOU, K. Osmoconditioning enhances cucumber and tomato seed germinability under adverse light conditions. Israel Journal of Botany, v.37, n.1, p.1-10, 1988.

TRIGO, M.F.O.O.; NEDEL, J.L.; TRIGO, L.F.N. Condicionamento osmótico em sementes de cebola: I. Efeitos sobre a germinação. Scientia Agricola, v.56, n.4, p.1059-1067, 1999.
TRIGO,M.F.O.O.;TRIGO,L.F.N.Efeitodocondicionamento osmótico na germinação e no vigor de sementes de berinjela (Solanum melongena L.). Revista Brasileira de Sementes, v.21, n.1, p.107-113, 1999.

WELBAUM, G.E.; SHEN, Z.; OLUOCH, M.O.; JETT, L.W. The evolution and effects of priming vegetable seeds. Seed Technology, v.20, n.2, p.209-235, 1998.

ZENGH, G.H.; WILEN, R.W.; SLINKARD, A.E.; GUSTA, L.V. Enhancement of canola seed germination and seedling emergence at low temperature by priming. Crop Science, v.34, n.5, p.1589-1593, 1994. 